\title{
A very general quartic double fourfold or fivefold is not stably rational
}

\author{
Arnaud Beauville
}

\begin{abstract}
Applying an idea of C. Voisin, we prove that a double cover of $\mathbb{P}^{4}$ or $\mathbb{P}^{5}$ branched along a very general quartic hypersurface is not stably rational.
\end{abstract}

\section{Introduction}

A projective variety $X$ is stably rational if $X \times \mathbb{P}^{m}$ is rational for some integer $m$. A stably rational variety is unirational; that the converse does not hold was shown by Artin and Mumford [AM72]. Their example is a double covering $X$ of $\mathbb{P}_{\mathbb{C}}^{3}$ branched along a quartic symmetroid, a surface defined by the vanishing of a symmetric 4 -by-4 determinant of linear forms. They prove that the torsion subgroup of $H^{3}(X, \mathbb{Z})$ is nonzero, whereas it is trivial for stably rational varieties.

Unfortunately this method applies only to rather particular varieties, and not to natural families like Fano threefolds, complete intersections, etc. A more powerful approach was discovered recently by Voisin [Voi15]: the existence of torsion in $H^{3}(X, \mathbb{Z})$ implies the nontriviality of a certain Chow group, a property which behaves better under specialization. She obtained the following beautiful consequence.

TheOrem (Voisin). A double cover of $\mathbb{P}_{\mathbb{C}}^{3}$ branched along a very general quartic surface is not stably rational.

Here "very general" means that the surface lies outside the union of countably many strict subvarieties in the space of quartic surfaces in $\mathbb{P}^{3}$.

The aim of this paper is to extend this result in higher dimension, as follows.

Theorem 1. For $n=4$ or 5 , a double cover of $\mathbb{P}_{\mathbb{C}}^{n}$ branched along a very general quartic hypersurface is not stably rational.

These varieties are easily seen to be unirational (Proposition 5); to our knowledge they provide the first examples of prime (that is, with Picard number 1) Fano manifolds of dimension greater than 3 which are unirational but not rational.

To prove Theorem 1 we apply Voisin's method, as extended in [CTP14]. The following is the statement that we will use ([CTP14, Théorème 1.12] and [Voi15, Remark 1.3]).

Received 9 December 2014, accepted in final form 8 March 2015.

2010 Mathematics Subject Classification 14E08 (primary), 14M20, 14J45 (secondary).

Keywords: unirational, stably rational, double coverings, linear systems of quadrics, Brauer group.

This journal is (c) Foundation Compositio Mathematica 2015. This article is distributed with Open Access under the terms of the Creative Commons Attribution Non-Commercial License, which permits non-commercial reuse, distribution, and reproduction in any medium, provided that the original work is properly cited. For commercial re-use, please contact the Foundation Compositio Mathematica. 


\section{QUARTIC DOUBLE 4- OR 5-FOLDS ARE NOT STABLY RATIONAL}

Proposition 1. Let $B$ be a smooth complex variety, let o be a closed point of $B$, and let $f: \mathcal{X} \rightarrow B$ be a flat, projective morphism such that the generic fiber of $f$ is smooth and that the fiber $X:=\mathcal{X}_{\mathrm{o}}$ is integral and admits a desingularization $\sigma: \tilde{X} \rightarrow X$ with the following properties:

a) The torsion subgroup of $H^{3}(\tilde{X}, \mathbb{Z})$ is non trivial.

b) The fiber of $\sigma$ over any point $x \in X$ is a smooth rational variety over the residual field $\kappa(x)$.

Then for a very general point $b \in B$, the fiber $\mathcal{X}_{b}$ is not stably rational.

We stress that condition b) must hold for all points of the scheme $X$, not only for closed points. Actually, Proposition 1 gives the (possibly) stronger result that $\mathcal{X}_{b}$ is not retract rational. Thus in Theorem 1 one can replace "stably rational" by "retract rational".

Voisin's theorem follows at once from Proposition 1 by taking for $X$ the Artin-Mumford example. To treat the higher-dimensional case, we simply take the obvious generalization of that example, namely a double covering $X \rightarrow \mathbb{P}^{n}$ branched along a quartic symmetroid. The variety $X$ is singular, but admits for $n=4$ or 5 a simple desingularization ${ }^{1}$ which satisfies condition b) of Proposition 1 (see Proposition 3). To check condition a), we view $\mathbb{P}^{n}$ as a linear system $L$ of quadrics in $\mathbb{P}^{3}$; then the smooth part $X_{\mathrm{sm}}$ of $X$ parametrizes the quadrics of $L$ of rank at least 3 together with the choice of a system of generatrices. The generatrices in each system are parametrized by $\mathbb{P}^{1}$, so we get a $\mathbb{P}^{1}$-bundle over $X_{\mathrm{sm}}$; this provides a 2 -torsion class in $H^{3}\left(X_{\mathrm{sm}}, \mathbb{Z}\right)$. We will prove that this class comes from a nontrivial torsion class in $H^{3}(\tilde{X}, \mathbb{Z})$ (Proposition 4 ), whence the result.

\section{Linear systems of quadrics}

2.1. Let $\mathcal{Q}$ be the linear system of quadrics in $\mathbb{P}_{\mathbb{C}}^{3}$. We denote by $\mathcal{Q}_{i} \subset \mathcal{Q}$ the subvariety of quadrics of rank at most $i$. We recall some basic properties of these varieties (see, for instance, [Vai82]):

- We have $\mathcal{Q} \cong \mathbb{P}^{9}$, the subvariety $\mathcal{Q}_{3}$ is a quartic hypersurface in $\mathcal{Q}$, $\operatorname{dim} \mathcal{Q}_{2}=6$, and $\operatorname{dim} \mathcal{Q}_{1}=3$.

- The singular locus of $\mathcal{Q}_{i}$ is $\mathcal{Q}_{i-1}$.

- The tangent cone $T C_{q}\left(\mathcal{Q}_{3}\right)$ at a point $q$ of $\mathcal{Q}_{2} \backslash \mathcal{Q}_{1}$ is a rank 3 quadric in $T_{q}(\mathcal{Q})$.

2.2. For $n=3,4$ or 5 , let $L$ be an $n$-dimensional projective subspace of $\mathcal{Q}$. We assume that $L$ does not meet $\mathcal{Q}_{1}$ and is transverse to $\mathcal{Q}_{2}$ - this is the case if $L$ is sufficiently general. We put

$$
\Delta:=L \cap \mathcal{Q}_{3} \quad \text { and } \quad \Sigma:=L \cap \mathcal{Q}_{2} .
$$

Thus $\Delta$ is a quartic hypersurface in $L$, with singular locus $\Sigma$ that is smooth, of dimension $n-3$. The tangent cone $T C_{q}(\Delta)$ at a point $q$ of $\Sigma$ is a rank 3 quadric in $T_{q}(L)$ (that is, a cone over a smooth conic, with vertex a linear space of dimension $n-3)$.

2.3. Let $b: \tilde{L} \rightarrow L$ be the blow-up of $L$ along $\Sigma$; let $E$ be the exceptional divisor, and let $\tilde{\Delta}$ be the strict transform of $\Delta$.

\footnotetext{
${ }^{1}$ The desingularization becomes more complicated for $n \geqslant 6$; see Subsection 5.1.
} 


\section{A. Beauville}

Proposition 2. The strict transform $\tilde{\Delta}$ is smooth and intersects $E$ transversally, so that $C:=$ $\tilde{\Delta} \cap E$ is smooth. Locally over $\Sigma$ for the Zariski topology, the embedding $C \hookrightarrow E$ is isomorphic to the embedding $C_{0} \times \Sigma \hookrightarrow \mathbb{P}^{2} \times \Sigma$, where $C_{0}$ is a smooth conic in $\mathbb{P}^{2}$.

Proof. The fibration $E \rightarrow \Sigma$ is the projectivization of the normal bundle $N(\Sigma / L)=T L_{\mid \Sigma} / T \Sigma$, while the fibration $C \rightarrow \Sigma$ is the projectivization of the normal cone $N C(\Sigma / \Delta)=T C(\Delta)_{\mid \Sigma} / T \Sigma$ (observe that at each point $q$ of $\Sigma$, the tangent space $T_{q} \Sigma$ is the vertex of the tangent cone $T C_{q}(\Delta)$ ). By Subsection 2.2, the fibration $C \rightarrow \Sigma$ is a smooth conic bundle. Since $C$ is a Cartier divisor in $\tilde{\Delta}$, this implies that $\tilde{\Delta}$ is smooth along $C$, and therefore everywhere.

There is another natural $\mathbb{P}^{1}$-bundle over $\Sigma$ : Let $C^{\prime} \subset \mathbb{P}^{3} \times \Sigma$ be the variety of pairs $(x, q)$ with $x \in \operatorname{Sing}(q)$. The projection $C^{\prime} \rightarrow \Sigma$ is a $\mathbb{P}^{1}$-bundle, with fiber $\operatorname{Sing}(q)$ above $q \in \Sigma$. It is easy to see that it is locally trivial for the Zariski topology. In fact, writing $\mathbb{P}^{3}=\mathbb{P}(V)$, we have a "universal quadric" $q_{L} \in H^{0}\left(L, \operatorname{Sym}^{2} V^{*} \otimes \mathcal{O}_{L}(1)\right)$ over $L$, or equivalently a symmetric map $q_{L}^{\sharp}: V \otimes \mathcal{O}_{L} \rightarrow V^{*} \otimes \mathcal{O}_{L}(1)$. The kernel of $q_{L \mid \Sigma}^{\sharp}$ is a rank 2 vector bundle $K$ on $\Sigma$, and we have $C^{\prime}=\mathbb{P}_{\Sigma}(K)$. We will now compare the $\mathbb{P}^{1}$-bundles $C$ and $C^{\prime}$.

The projective tangent cone $\mathbb{P} T C_{q}(\Delta)$ to $\Delta$ at a singular point $q$ can be viewed as the variety of lines in $L$ passing through $q$ and intersecting $\Delta$ with multiplicity at least 3 . Let $r \in L$; we denote by $\dot{q}$ and $\dot{r}$ quadratic forms defining $q$ and $r$, respectively. The line $\langle q, r\rangle$ belongs to $\mathbb{P T C}_{q}(\Delta)$ if and only if $\operatorname{det}(\dot{q}+t \dot{r})$ is divisible by $t^{3}$. Choose a decomposition $V=W \oplus \operatorname{Sing}(q)$. Then $\dot{q}+t \dot{r}$ is represented by a block matrix

$$
\left(\begin{array}{c|c}
\dot{q}_{\mid W}+t \dot{r}_{\mid W} & t(\ldots) \\
\hline t(\ldots) & t \dot{r}_{\mid \operatorname{Sing}(q)}
\end{array}\right),
$$

with $\operatorname{det}\left(\dot{q}_{\mid W}\right)=\lambda \neq 0$. Thus $\operatorname{det}(\dot{q}+t \dot{r})=t^{2} \lambda \operatorname{det}\left(\dot{r}_{\mid \operatorname{Sing}(q)}\right)\left(\bmod t^{3}\right)$, so the above condition is equivalent to $\operatorname{det}\left(\dot{r}_{\mid \operatorname{Sing}(q)}\right)=0$, that is, to the quadric $r$ being tangent to $\operatorname{Sing}(q)$.

Similarly, the line $\langle q, r\rangle$ belongs to $\mathbb{P} T_{q}(\Sigma)$ if and only if all 3-by-3 minors of $\dot{q}+t \dot{r}$ are divisible by $t^{2}$; this is equivalent to $r$ containing the line $\operatorname{Sing}(q)$. Thus we have a canonical identification of the projectivization of the normal cone $T C_{q}(\Delta) / T_{q}(\Sigma)$ with $\operatorname{Sing}(q)$, mapping a line $\langle q, r\rangle$ not tangent to $\Sigma$ to the point of contact of $r$ with $\operatorname{Sing}(q)$. This shows that the $\mathbb{P}^{1}$-bundle $C$ is isomorphic to $C^{\prime}$, hence locally trivial for the Zariski topology.

Finally, put $N:=N(\Sigma / L)$; let $p$ be the projection $C \rightarrow \Sigma$. The embedding $i: C \hookrightarrow E=$ $\mathbb{P}_{\Sigma}(N)$ is determined by the line bundle $M:=i^{*} \mathcal{O}_{E}(1)$ and the surjective homomorphism $p^{*} N^{*} \rightarrow M$. The latter gives by adjunction an isomorphism $N^{*} \stackrel{\sim}{\longrightarrow} p_{*} M$, so $i$ is isomorphic to the embedding $C \hookrightarrow \mathbb{P}_{\Sigma}\left(\left(p_{*} M\right)^{*}\right)$.

Let $q \in \Sigma$. Replacing $\Sigma$ by a Zariski open subset containing $q$, we may assume that $p$ is the projection $\mathbb{P}^{1} \times \Sigma \rightarrow \Sigma$ and that $M$ is the pull-back of $\mathcal{O}_{\mathbb{P}^{1}}(2)$. Then $p_{*} M \cong \mathcal{O}_{\Sigma}^{3}$, and $i$ is isomorphic to the embedding $C_{0} \times \Sigma \hookrightarrow \mathbb{P}^{2} \times \Sigma$ in a Zariski neighborhood of $q$.

\section{The double covering}

Let $\pi: X \rightarrow L$ be the double covering of $L$ branched along the quartic hypersurface $\Delta$. The variety $X$ is singular along $\pi^{-1}(\Sigma)$. The class $b^{*}(\Delta)-2 E$ of $\tilde{\Delta}$ in $\operatorname{Pic}(\tilde{L})$ is divisible by 2 , hence we can form the double covering $\tilde{X} \rightarrow \tilde{L}$ branched along $\tilde{\Delta}$. It gives a resolution $\sigma: \tilde{X} \rightarrow X$ of $X$, which is an isomorphism outside $\Sigma$; the variety $Q:=\sigma^{-1}(\Sigma)$ is a double covering of $E$ branched along $C$. 


\section{QUARTIC DOUBLE 4- OR 5-FOLDS ARE NOT STABLY RATIONAL}

Proposition 3. The resolution $\sigma$ induces a smooth quadric fibration $Q \rightarrow \Sigma$, locally trivial for the Zariski topology. In particular, for any $q \in \Sigma$ the fiber $\sigma^{-1}(q)$ is a smooth quadric, rational over $\kappa(q)$.

Proof. Let $q \in \Sigma$. In view of Proposition 2, replacing $\Sigma$ by a Zariski open subset containing $q$, we may assume that $Q$ is a double covering of $\mathbb{P}^{2} \times \Sigma$ branched along $C_{0} \times \Sigma$. Such a double covering is determined by the branch locus $C_{0} \times \Sigma$ and a line bundle $M$ on $\mathbb{P}^{2} \times \Sigma$ such that $M^{\otimes 2} \cong \operatorname{pr}_{1}^{*} \mathcal{O}_{\mathbb{P}^{2}}(2)$. Shrinking $\Sigma$ again, we may assume $M \cong \operatorname{pr}_{1}^{*} \mathcal{O}_{\mathbb{P}^{2}}(1)$; then the covering $Q \rightarrow \mathbb{P}^{2} \times \Sigma$ is isomorphic to $Q_{0} \times \Sigma \rightarrow \mathbb{P}^{2} \times \Sigma$, where $Q_{0} \rightarrow \mathbb{P}^{2}$ is the double covering of $\mathbb{P}^{2}$ branched along $C_{0}$. Since $Q_{0}$ is a smooth quadric, this implies the proposition.

This gives us condition b) of Proposition 1; we now check condition a).

Proposition 4. The 2-torsion subgroup of $H^{3}(\tilde{X}, \mathbb{Z})$ is nontrivial.

Proof. We will prove that the Brauer group $\operatorname{Br}(\tilde{X})$ [Gro68] contains a nonzero 2-torsion element. This group injects into $H^{2}\left(\tilde{X}, \mathcal{O}_{h}^{*}\right)$, where $\mathcal{O}_{h}$ is the sheaf of holomorphic functions on $\tilde{X}$. Since $H^{2}\left(\tilde{X}, \mathcal{O}_{\tilde{X}}\right)=0$, the latter group injects into $H^{3}(\tilde{X}, \mathbb{Z})$ by the exponential exact sequence, so this implies the proposition.

We put $U:=\tilde{X} \backslash Q$. Let $\mathbb{G}:=\mathbb{G}(2,4)$ denote the Grassmannian of lines in $\mathbb{P}^{3}$. We consider the incidence variety $I:=\{(\ell, q) \in \mathbb{G} \times(L \backslash \Sigma) \mid \ell \subset q\}$ and the projection $p: I \rightarrow L \backslash \Sigma$.

The fiber $p^{-1}(q)$ is a disjoint union of two rational curves for $q \in L \backslash \Delta$ and is a single rational curve for $q \in \Delta \backslash \Sigma$. Therefore $p$ factors as a $\mathbb{P}^{1}$-fibration $\varphi: I \rightarrow U$, followed by the double covering $U \rightarrow L \backslash \Sigma$. The $\mathbb{P}^{1}$-bundle $\varphi$ gives a 2 -torsion class $[\varphi]$ in the Brauer group $\operatorname{Br}(U)$ [Gro68]. We claim that this class is nonzero.

Suppose first $n=3$. If the class of $\varphi$ in $\operatorname{Br}(U)$ were zero, the $\mathbb{P}^{1}$-bundle $\varphi: I \rightarrow U$ would be a projective bundle. But $I$ is a rational variety, because the projection $I \rightarrow \mathbb{G}$ is birational [Bea83, $\S 9$, and we know that $\tilde{X}$ is not stably rational [AM72]. We conclude that $[\varphi] \neq 0$ in $\operatorname{Br}(U)$.

For $n=4$ or 5 , we choose a general 3-dimensional projective subspace $L^{\prime}$ in $L$, and construct the corresponding subvarieties $U^{\prime} \subset U$ and $I^{\prime}:=\varphi^{-1}\left(U^{\prime}\right)$. The class $[\varphi]$ in $\operatorname{Br}(U)$ restricts to $\left[\varphi_{\mid I^{\prime}}\right]$ in $\operatorname{Br}\left(U^{\prime}\right)$, which is nonzero by the above; thus we find $[\varphi] \neq 0$ in $\operatorname{Br}(U)$ in all cases. We conclude with the following lemma.

LEMmA. The restriction map $\operatorname{Br}(\tilde{X}) \rightarrow \operatorname{Br}(U)$ is an isomorphism.

Proof. We consider the quadric fibration $f: Q \rightarrow \Sigma$. The two systems of generatrices of each fiber form a double covering of $\Sigma$ which is locally trivial for the Zariski topology (Proposition 3), hence trivial. We choose one of the two systems. In each fiber the generatrices of this system are parametrized by $\mathbb{P}^{1}$ and form a $\mathbb{P}^{1}$-fibration $g: G \rightarrow \Sigma$. For each point $x$ of $Q$ there is a unique generatrix of our system passing through $x$; this gives again a $\mathbb{P}^{1}$-fibration $h: Q \rightarrow G$ such that $g \circ h=f$. By Proposition 3 both fibrations are locally trivial for the Zariski topology, hence are projective bundles.

We claim that we can blow down $\tilde{X}$ along the fibers of $h$, more precisely, that there exist a compact complex manifold $\bar{X}$, a map $p: \tilde{X} \rightarrow \bar{X}$ and an embedding $G \hookrightarrow \bar{X}$ such that the diagram

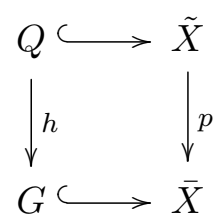




\section{A. Beauville}

is obtained by blowing up $G$ in $\bar{X}$. According to the Fujiki-Nakano criterion [FN71], it suffices to prove that the restriction of the line bundle $\mathcal{O}_{\tilde{X}}(Q)$ to a fiber $\ell:=h^{-1}(q)$ of $h$ has degree -1 .

We have $K_{Q \mid \ell} \cong K_{Q_{q \mid \ell}} \cong \mathcal{O}_{\ell}(-2)$. Recall that $\tilde{X}$ was obtained by first taking the blowup $b: \tilde{L} \rightarrow L$ of $L$ along $\Sigma$, with exceptional divisor $E$, and then taking the double covering $d: \tilde{X} \rightarrow \tilde{L}$ branched along the surface $\tilde{\Delta} \in\left|b^{*} \Delta-2 E\right|$. Then

$$
K_{\tilde{L}} \cong b^{*} \mathcal{O}_{L}(-n-1)(2 E) \text { and } K_{\tilde{X}} \cong d^{*} b^{*} \mathcal{O}_{L}(-n+1)(Q)
$$

since $\ell$ is contracted by $b \circ d$, we find $K_{\tilde{X} \mid \ell} \cong \mathcal{O}_{\tilde{X}}(Q)_{\mid \ell}$. Using the adjunction formula we get $K_{Q \mid \ell} \cong K_{\tilde{X}}(Q)_{\mid \ell} \cong \mathcal{O}_{\tilde{X}}(2 Q)_{\mid \ell}$, therefore $\operatorname{deg} \mathcal{O}_{\tilde{X}}(Q)_{\mid \ell}=-1$, whence our claim.

Now, we have a commutative diagram

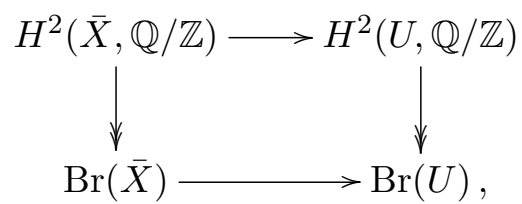

where the vertical arrows are surjective; the top horizontal arrow is bijective by the Gysin exact sequence, because $G$ has codimension 2 in $\bar{X}$. Therefore the restriction map $\operatorname{Br}(\bar{X}) \rightarrow \operatorname{Br}(U)$ is surjective. Since it is the composition of $p^{*}: \operatorname{Br}(\bar{X}) \rightarrow \operatorname{Br}(\tilde{X})$ and the restriction map $\operatorname{Br}(\tilde{X}) \rightarrow$ $\operatorname{Br}(U)$, it follows that the latter is surjective; but it is also injective since both Brauer groups inject into the Brauer group of the function field $\mathbb{C}(\tilde{X})$; see [Gro68, II, Corollaire 1.10].

Thus our desingularization $\tilde{X} \rightarrow X$ satisfies the conditions stated in Proposition 1. Theorem 1 follows by taking for $B$ the space of quartic hypersurfaces in $L=\mathbb{P}^{n}$, for o $\in B$ the point corresponding to $\Delta$, and for $\mathcal{X}$ the family of double coverings of $\mathbb{P}^{n}$ branched along those hypersurfaces.

\section{Unirationality}

The following result is classical for $n=3$, and the proof extends easily to the general case.

Proposition 5. A double covering of $\mathbb{P}^{n}$ branched along an integral quartic hypersurface is unirational.

Proof. Let $\pi: X \rightarrow \mathbb{P}^{n}$ be the double covering, and let $\mathbb{G}$ be the Grassmannian of lines in $\mathbb{P}^{n}$. Consider the variety $X^{*} \subset X \times \mathbb{G}$ of pairs $(x, \ell)$ with $\pi(x) \in \ell$. The projection $p: X^{*} \rightarrow X$ is a projective $\mathbb{P}^{n-1}$-bundle, with fiber at $x \in X$ the space of lines passing through $\pi(x)$.

For $(x, \ell)$ general in $X^{*}$, the curve $E:=\pi^{-1}(\ell)$ is a smooth genus 1 curve in $X$ passing through $x$; there is a unique point $f(x, \ell) \in E$ such that the divisors $\pi^{*} q+f(x, \ell)$, for $q \in \ell$, are linearly equivalent to $3 x$. This defines a rational map $f: X^{*} \rightarrow X$.

Let $(y, \ell)$ be a general point of $X^{*}$. On the genus 1 curve $\pi^{-1}(\ell)$ there are nine points $x$ such that $3 x$ is linearly equivalent to $\pi^{*} q+y$ for $q \in \ell$, that is, such that $f(x, \ell)=y$. Thus $f$ is dominant, and a general fiber $f^{-1}(y)$ has dimension $n-1$; in particular, we have $p\left(f^{-1}(y)\right) \varsubsetneqq X$.

Let $P$ be a general 2-plane in $\mathbb{P}^{n}$, and let $\tilde{P}:=\pi^{-1}(P) \subset X$. We consider the restriction $f_{P}$ of $f$ to $p^{-1}(\tilde{P})$. We have $p\left(f_{P}^{-1}(y)\right)=\tilde{P} \cap p\left(f^{-1}(y)\right) \varsubsetneqq \tilde{P}$. The projection $p: f_{P}^{-1}(y) \rightarrow \tilde{P}$ is generically injective (if $f(x, \ell)=y$, then $\ell=\langle\pi(x), \pi(y)\rangle$ ). Thus $\operatorname{dim} f_{P}^{-1}(y) \leqslant 1$. It follows that $f_{P}: p^{-1}(\tilde{P}) \rightarrow X$ is dominant. But $p^{-1}(\tilde{P})$ is a projective bundle over the rational surface $\tilde{P}$, hence is rational, and $X$ is unirational. 


\section{QUARTIC DOUBLE 4- OR 5-FOLDS ARE NOT STABLY RATIONAL}

\section{Questions}

5.1. It might be possible to extend our main result in dimension $n=6, \ldots, 9$, by taking a general linear system $L \subset \mathcal{Q}$ of dimension $n$. However, for $n \geqslant 6$ this linear system contains rank 1 quadrics, which produce triple points of $\Delta$. The desingularization becomes much more intricate; we do not know whether the conditions a) and b) of Proposition 1 still hold.

5.2. In [CTP14] the authors show that a very general quartic threefold is not stably rational, by applying Proposition 1 to a singular quartic birational to the Artin-Mumford threefold. This has been extended by Totaro [Tot15] to very general hypersurfaces of degree at least $2\lceil(n+2) / 3\rceil$ in $\mathbb{P}^{n+1}$, in particular to quartic fourfolds, by combining Proposition 1 with an earlier method of Kollár. It would be interesting to extend the result to very general quartic fivefolds, which are known to be unirational [CM98].

\section{ACKNOWLEDGEMENTS}

I am grateful to Claire Voisin for a very useful discussion, and to the referee for his or her careful reading, in particular for simplifying the proof of Proposition 4.

\section{REFERENCES}

AM72 M. Artin and D. Mumford, Some elementary examples of unirational varieties which are not rational, Proc. London Math. Soc. (3) 25 (1972), 75-95; http://dx.doi.org/10.1112/plms/ s3-25.1.75.

Bea83 A. Beauville, Variétés rationnelles et unirationnelles, Algebraic Geometry - Open Problems (Ravello, 1982), Lecture Notes in Math., vol. 997 (Springer, Berlin, 1983), 16-33; http://dx. doi.org/10.1007/BFb0061635.

CM98 A. Conte and J.P. Murre, On a theorem of Morin on the unirationality of the quartic fivefold, Atti Accad. Sci. Torino Cl. Sci. Fis. Mat. Natur. 132 (1998), 49-59.

CTP14 J.-L. Colliot-Thélène and A. Pirutka, Hypersurfaces quartiques de dimension 3: non rationalité stable, Ann. Sci. École Norm. Sup., to appear, arXiv:1402.4153.

FN71 A. Fujiki and S. Nakano, Supplement to "On the inverse of monoidal transformation", Publ. Res. Inst. Math. Sci. 7 (1971), no. 3, 637-644; http://doi.org/10.2977/prims/1195193401.

Gro68 A. Grothendieck, Le groupe de Brauer I, II, in Dix exposés sur la cohomologie des schémas (North-Holland, Amsterdam; Masson, Paris, 1968), 46-87.

Tot15 B. Totaro, Hypersurfaces that are not stably rational, J. Amer. Math. Soc., to appear, arXiv:1502.04040; http://dx.doi.org/10.1090/jams/840.

Vai82 I. Vainsencher, Schubert calculus for complete quadrics, Enumerative Geometry and Classical Algebraic Geometry (Nice, 1981), Progr. Math., vol. 24 (Birkhäuser, Boston, MA, 1982), 199235; http://dx.doi.org/10.1007/978-1-4684-6726-0_10.

Voi15 C. Voisin, Unirational threefolds with no universal codimension 2 cycle, Invent. Math. 201 (2015), no. 1, 207-237; http://dx.doi.org/10.1007/s00222-014-0551-y.

Arnaud Beauville arnaud.beauville@unice.fr

Laboratoire J.-A. Dieudonné, UMR 7351 du CNRS, Université de Nice, Parc Valrose, 06108 Nice cedex 2, France 